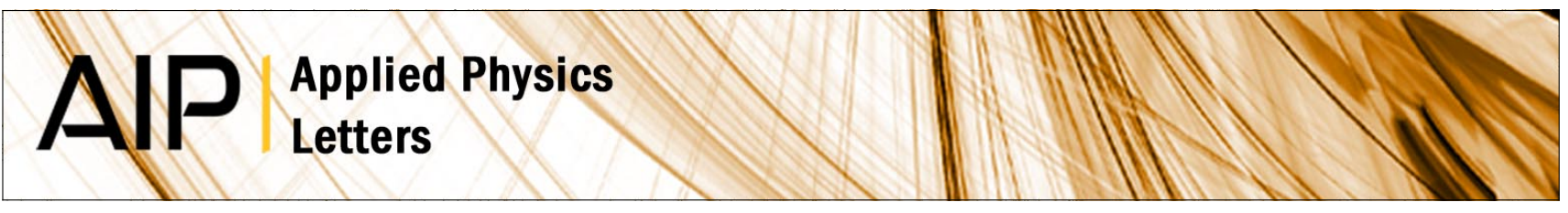

Microwave surface waves supported by a tapered geometry metasurface

Elizabeth M. G. Brock and Alastair P. Hibbins

Citation: Appl. Phys. Lett. 103, 111904 (2013); doi: 10.1063/1.4820411

View online: http://dx.doi.org/10.1063/1.4820411

View Table of Contents: http://apl.aip.org/resource/1/APPLAB/v103/i11

Published by the AIP Publishing LLC.

Additional information on Appl. Phys. Lett.

Journal Homepage: http://apl.aip.org/

Journal Information: http://apl.aip.org/about/about_the_journal

Top downloads: http://apl.aip.org/features/most_downloaded

Information for Authors: http://apl.aip.org/authors

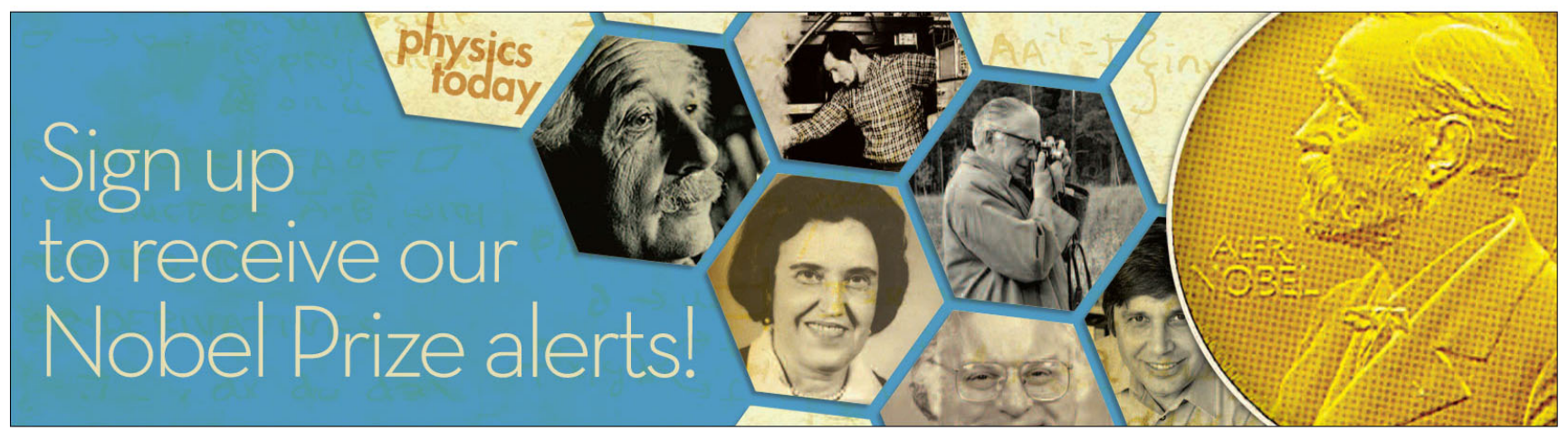




\title{
Microwave surface waves supported by a tapered geometry metasurface
}

\author{
Elizabeth M. G. Brock ${ }^{\text {a) }}$ and Alastair P. Hibbins \\ Electromagnetic and Acoustic Materials Group, Department of Physics and Astronomy, University of Exeter, \\ Exeter, Devon EX4 4QL, United Kingdom
}

(Received 30 May 2013; accepted 22 August 2013; published online 10 September 2013)

\begin{abstract}
Spatial dependent reduction of a surface wave's group velocity is demonstrated in the microwave regime across a metasurface comprised linear array of rectangular cavities. We manipulate the surface wave dispersion through variation in cavity width to provide a spatially dependent modal index. The mode is slowed and trapped at different positions along the metasurface. This "slow-light" phenomenon is observed via local electric-field and phase measurements. The latter is shown to provide a more accurate identification of the trapping location. We discuss the reflectivity of the mode close to this point and explore the excitation of higher order surface modes. (C) 2013 AIP Publishing LLC. [http://dx.doi.org/10.1063/1.4820411]
\end{abstract}

The prospect of slowing and completely stopping light is an attractive focus for many areas of physics ${ }^{1}$ due to the number of technological applications that would benefit from such control. The characteristic that defines slow light is a reduced group velocity, $v_{\mathrm{g}}=\mathrm{d} \omega / \mathrm{d} k<\mathrm{c}$, where $\omega$ and $k$ are the angular frequency and wavevector of the electromagnetic mode, respectively, and $\mathrm{c}$ is the speed of light. A seminal example of slowing light to $17 \mathrm{~ms}^{-1}$ using electromagnetic induced transparency (EIT) has been achieved by Hau et $a l^{2}$ over a very narrow bandwidth. Other methods include guiding a transverse electromagnetic mode along waveguides composed of different insulator-metal combinations or guides with a negative-index core clad in dielectric (insulator-negative-index-insulator) design ${ }^{3-7}$ or indeed through photonic crystal (PhC) waveguides. ${ }^{8-13}$ The total thickness of such slow light waveguide structure is of the same scale as the wavelength of light itself and therefore larger than the electrical components required to connect to it, limiting minimum geometric size. However, surface plasmons polaritons (SPPs) ${ }^{14}$ have provided a route to circumnavigate this limitation. SPPs are a collective excitation of free electrons at the interface between a metal and a dielectric, oscillating longitudinally and in coherence with incident visible light. They are intrinsically slow transverse-magnetic (TM) polarised modes, whose electromagnetic fields exponentially decay into each bounding medium. Importantly, the mode can be manipulated on subwavelength structures, an aspect which has recently been exploited in slow wave devices. ${ }^{14-19}$

Strictly, any electromagnetic mode cannot be completely stopped before Ohmic losses become dominant, ${ }^{20}$ an example of which is an SPP supported by a Drude like metal. However, it has been shown that a somewhat analogous mode to the SPP, with a similar dispersion, can be supported at microwave frequencies on metals patterned with subwavelength features. ${ }^{21}$ Such structures are known as metasurfa$\mathrm{ces}^{22}$ and can be described as a single layer of periodically arranged subwavelength elements arranged in a dielectric host which gain their electromagnetic properties from geometric design and not their constituent materials. Without such surface structure a metal would be unable to support a

a)emgb202@ex.ac.uk confined microwave surface wave ${ }^{23}$ due to its near-perfectly conducting ${ }^{24}$ nature in this frequency regime.

The collective resonant frequency of these metallic elements is synonymous with the surface plasma frequency of the aforementioned case because it is this limiting frequency, below which the necessary boundary condition (inductive surface impedance ${ }^{25}$ ) is induced for TM-polarised surface waves to be supported. At frequencies much lower than this limit, the surface wave is photon-like; however, on approach to this limiting frequency it becomes highly localised to the metasurface, and its group velocity reduces to zero. ${ }^{26}$ Such behaviour has been experimentally demonstrated on metasurfaces for a range of geometries, including open-ended grooves, ${ }^{26}$ holes, ${ }^{27,28}$ and Sievenpiper mushrooms. ${ }^{29}$

In this study, we experimentally demonstrate the spatial separation of frequency components associated with a microwave surface wave supported on a tapered metasurface. This slow-wave structure is composed of a linear array of constant depth, closed-sided air-filled rectangular cavities obtruding from a planar metal sheet. Two samples of this description are fabricated with their lateral widths tapering from $L_{\text {Start }}$ to $L_{\text {Finish }}$ (where $L_{\text {Start }} \ll L_{\text {Finish }}$ ), the dimensions of which are schematically depicted in Fig. 1. By increasing the lateral width of each cavity as a function of distance from the point of excitation, we modify the dispersion of the surface wave by lowering its limiting frequency. In this way, frequency components of the surface wave are slowed (due to an increase of their modal index, $n=c / v_{\mathrm{g}}$ ) and consequently trapped so that they cease to propagate further along the array. We demonstrate that local electric-field observations alone are not sufficient to determine the trapping point of the mode; however, utilisation of the local phase provides a more accurate method to probe this condition.

A vertically orientated antenna $(2.5 \mathrm{~mm}$ of unshielded core) provides near-field excitation of the surface wave. It is attached to port 1 of a vector network analyser (VNA) via a coaxial cable and is driven between the frequencies of $8-40 \mathrm{GHz}$. The probe remains in a static position throughout the experiment at $x=-289 \mathrm{~mm}, y=0 \mathrm{~mm}$ (mid-width of the array), and height $z=0.25 \mathrm{~mm}$ (above the obtruding metallic cavities). A second identical probe is also positioned at the same coordinates in $y$ and $z$ but is scanned along the length 


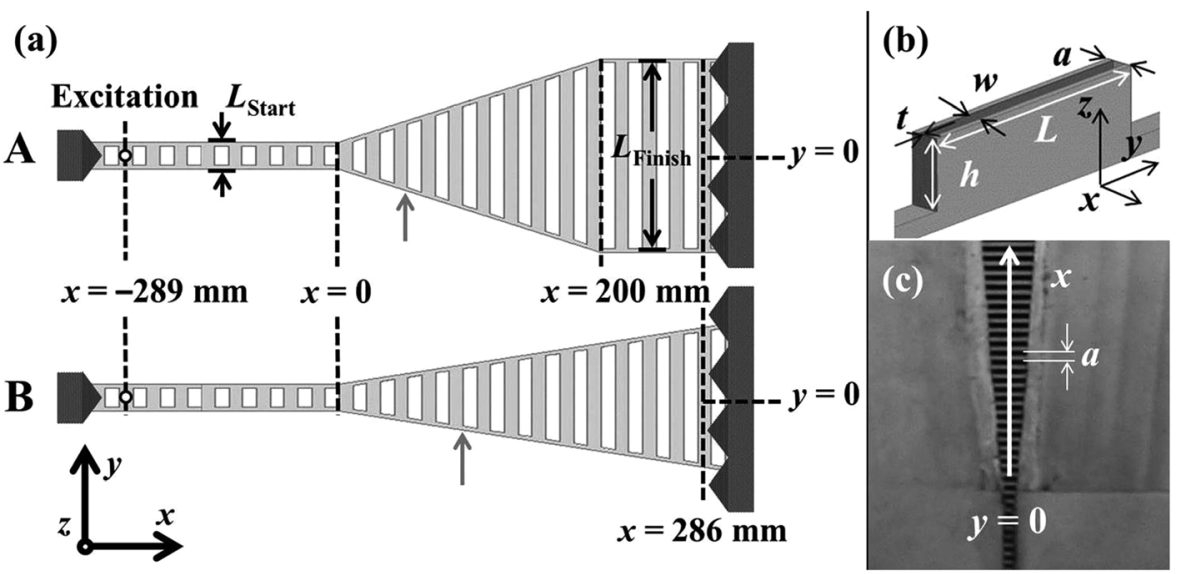

FIG. 1. (a) Schematic of samples A (top) and B (bottom) as viewed from above (white regions detailing internal structure are for illustrative purposed only). On both samples the tapering begins at $x=0$ with lateral width $L_{\text {Start }}=1.6 \mathrm{~mm}$. The taper ends with $L_{\text {Finish }}=48.5 \mathrm{~mm}$ at $x=200 \mathrm{~mm}$ for sample A, and $x=399 \mathrm{~mm}$ for sample B with $L_{\text {Finish }}=50 \mathrm{~mm}$. The position of the coaxial probe providing near field excitation is also shown. Microwave foam absorber is used to prevent reflections at each end $(x=-297 \mathrm{~mm}$ and $x=286 \mathrm{~mm})$ of samples A and B. The small grey arrows indicate the $x$-position on the sample, where $L=16.8 \mathrm{~mm}$. (b) A schematic illustration of a unit cell of the samples, where $L$ is the lateral width of the cavities, the cavity height $h=3.75 \mathrm{~mm}$, wall thickness $t=0.1 \mathrm{~mm}$, periodicity $a=1.59 \mathrm{~mm}$, and cavity width $w=a / 2$. (c) An photograph of a small section of the sample as viewed from above; the position of the white arrow indicates where $y=0$ along the $x$-direction.

of the array $(x)$ via operation of a computer controlled motorised stage. The local electric field amplitude and phase of the surface wave are detected via connection of this second probe to port 2 of the VNA.

In order to fully understand the slow light phenomenon associated with the surface wave excited on tapered samples A and B, it is first useful to study the dispersion of surface modes associated with one-dimensional periodic array of rectangular cavities with uniform widths (Fig. 2). Consider, for example, the regions defined by $x \in[-297,0]$ and $x \in$ [200, 285.25] (in units of $\mathrm{mm}$ ) along sample A, which have a lateral width $(L)$ of 1.6 and $48.5 \mathrm{~mm}$, respectively. In both of these regions the width is constant, and hence the modal index $(n)$ of the supported surface wave is dependent on frequency only. The surface mode dispersion is asymptotic to a frequency, to a first approximation, dictated by the lowest

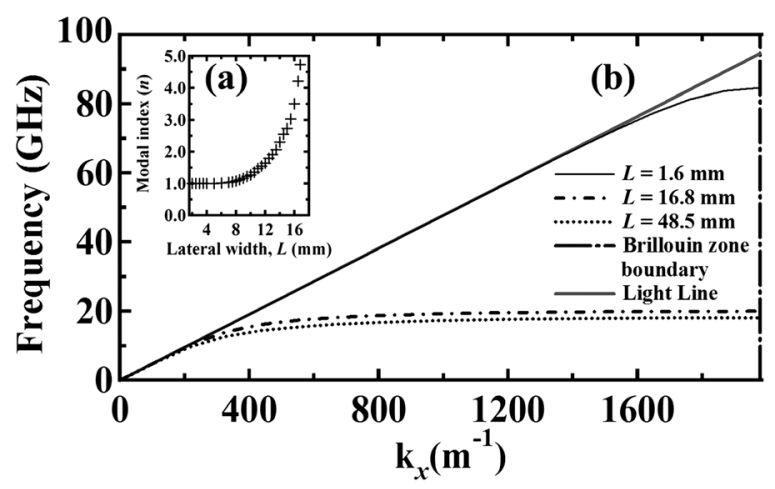

FIG. 2. Predicted dispersion of surface wave supported by a linear array of identical cavities of height $h=3.75 \mathrm{~mm}$, periodicity $a=1.59 \mathrm{~mm}$, and cavity width $w=a / 2$ in instances of $L=1.6,16.8$, and $48.5 \mathrm{~mm}$. Inset: predicted modal index of the supported wave at different lateral widths $L$ for $20.0 \mathrm{GHz}$. These predictions have been obtained via FEM modelling ${ }^{33}$ to find the eigenmodes of a unit cell of the structure bounded by phase-linked periodic boundary conditions ( $x z$-planes) to set $k_{\mathrm{x}}$. Since the modes of interest are TM polarised, and the unit cell is infinite in the $y$-direction, perfectmagnetic boundary conditions are utilised on $y z$-planes to limit the unit cell dimension. The model geometry is bounded $45 \mathrm{~mm}$ above the metasurface by a free space boundary. The predictions of modal index, $n=c / v_{\mathrm{g}}$. order Fabry-Perot resonance of the transverse electric $\left(\mathrm{TE}_{01}\right)$ waveguide mode supported in the cavity. ${ }^{27,30-32}$ The exact limiting frequency is numerically determined via finite element method $\left(\mathrm{FEM}^{33}\right)$ modelling for arrays of constant width $L=1.6 \mathrm{~mm}, 16.8 \mathrm{~mm}$, and $48.5 \mathrm{~mm}$ to be $84.6 \mathrm{GHz}$, $20.0 \mathrm{GHz}$, and $16.4 \mathrm{GHz}$, respectively (Fig. 2), i.e., we would expect the $20 \mathrm{GHz}$ component of the surface mode to have $v_{\mathrm{g}}=0$ and be trapped when $L=16.8 \mathrm{~mm}$.

Far below each limiting frequency, the surface mode is photon-like (i.e., lies close to light line), with a modal index $n \sim 1$. However, the surface mode supported by the tapered array propagates from the narrow width region (higher limiting frequency) to the wider width region (lower limiting frequency). Therefore the modal index is dependent on both frequency and $x$-position along the sample. Consequently, frequency components of the surface wave between these limits will be spectrally separated and prevented from propagating further with the highest frequencies being slowed and trapped closest to the start of the taper. Further to this, we consider two samples with different gradients of taper $(\Delta L / \Delta x)$ and contrast the reflection of the surface mode in each case.

Fig. 3 illustrates measurements of local amplitude and phase of the electric field as a function of position in $x$ along samples A and B at 20, 25, and $30 \mathrm{GHz}$. Note that the VNA measures local phase by sampling the fields of the detected surface wave and excitation source. By comparing these values a local phase difference is obtained, limited to the range $\pi$ to $-\pi$ radians, which is periodic in $x$ and indicative of the surface mode wavelength $\lambda_{\mathrm{SW}}=\lambda_{0} / n$, where $\lambda_{0}$ is the excitation wavelength.

Consider the global peak in the field amplitude data at $x=43 \mathrm{~mm}$ for sample A, and $x=77 \mathrm{~mm}$ for sample B (Figs. $3(a)$ and 3(b)). These peaks are indicative of an increase in the energy density of the surface mode associated with a reduction in its group velocity as the sample is traversed. Naively, these peaks may be viewed as an indication of the positions along the array at which the surface mode is trapped, ${ }^{34}$ after which it is no longer supported. However, it 

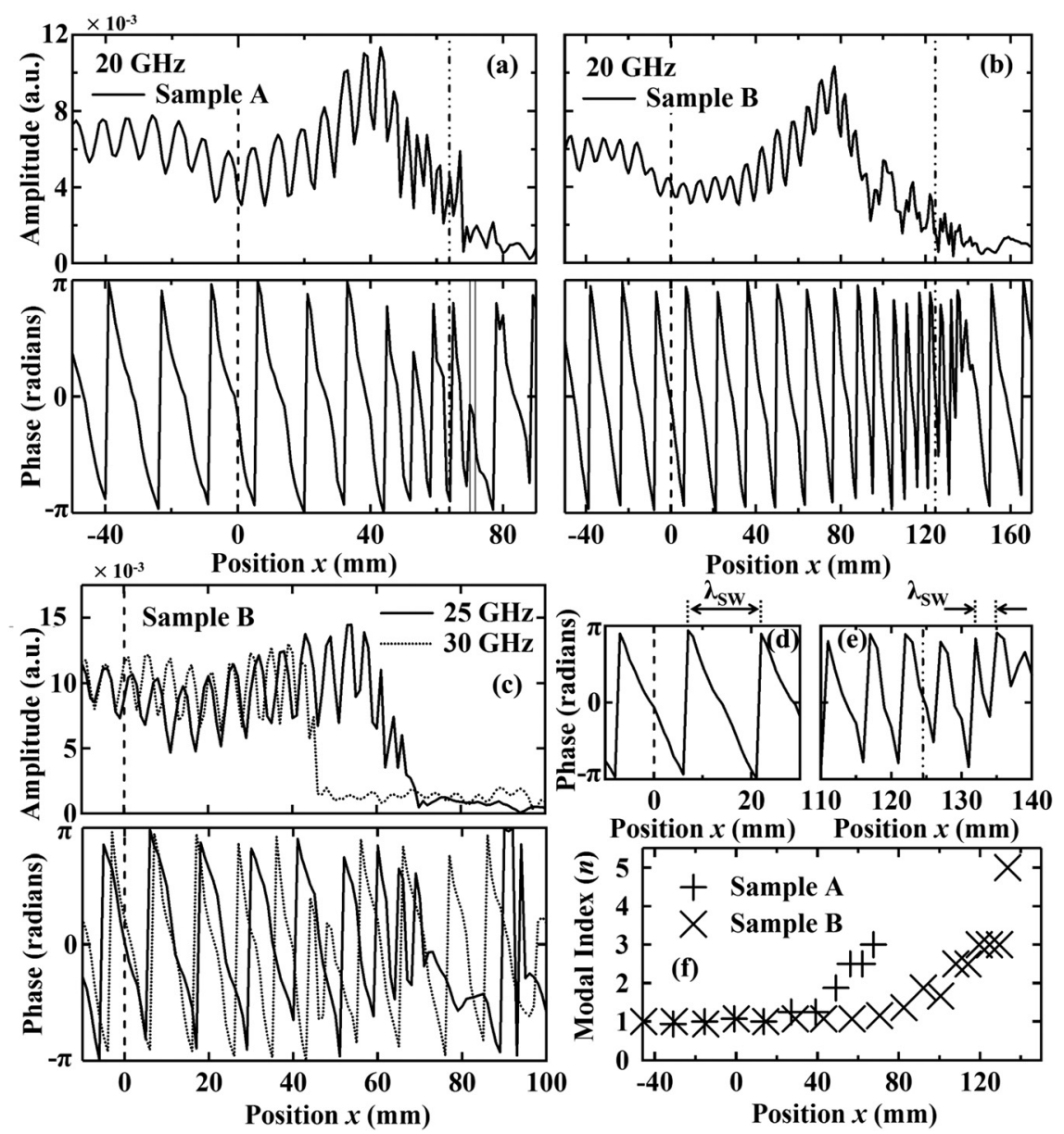

FIG. 3. Experimental observations of local field amplitude and phase of the surface wave at fixed frequencies as a function of distance $x$ along samples A and B. Vertical black dashed line indicates the position of the start of the taper $\left(L=L_{\text {Start }}\right)$. (a) Measurement at 20.0 GHz; doted-dashed line indicates the $x$-position of $L=16.8 \mathrm{~mm}$ along sample A where, for a onedimensional array of fixed width cavities, $20.0 \mathrm{GHz}$ is the limiting frequency. This position is indicated by the grey arrow in Fig. 1. (b) is the same for sample B. (c) Measurement at 25 and $30 \mathrm{GHz}$ of the mode supported by sample B. (d), (e) Closer inspection of (b), highlighting the periodicity of oscillation in recorded phase corresponding to surface wave wavelength $\left(\lambda_{\mathrm{SW}}\right)$ supported on two regions in $x$ along sample B at $20 \mathrm{GHz}$. (f) Experimentally derived modal index as a function of distance along samples A and $\mathrm{B}$ at $20 \mathrm{GHz}$ illustrating an increasing mode index (decreasing group velocity) with reduction of the asymptotic frequency. For both samples the surface mode is supported at $20 \mathrm{GHz}$ until the width of the supporting structure becomes $L=18.0 \mathrm{~mm}$. Thin vertical black lines on the lower part of (a) indicate the spatial extent of the pitch. should be remembered that the increase in the modal index (and propagation constant, $k_{\mathrm{x}}$ ) is accompanied by a reduction in the decay length $\left(\propto 1 / k_{\mathrm{z}}\right)$ of the mode above the surface, in order to conserve momentum. It is therefore clear that any measurement of the field magnitude at a fixed height above the sample $(0.25 \mathrm{~mm}$ in our case $)$ will be unable to accurately probe the exact trapping location of a surface wave. After this peak, the locally measured field amplitude gradually falls to zero, indicative that the mode eventually ceases to propagate $^{20,34,35}$ due to a combination of Bragg diffraction from the periodicity of the array and dissipative loss into the metal. Subsequently, the exact trapping location of the surface wave is ambiguous as this zero condition occurs at some distance after the field peak. However, by measuring the local phase of the surface wave, this condition can be more accurately observed and commented upon, as can the increasing modal index of the surface wave on approach to this location.

It is clear from the periodicity of the oscillation in the phase data in Figs. 3(a) and 3(b) that $\lambda_{\mathrm{SW}}$ has a value that is associated with the free space wavelength $\left(\lambda_{0}=15 \mathrm{~mm}\right.$ at $20 \mathrm{GHz}$ ) at $x=0$, where $L=L_{\text {Start }}=1.6 \mathrm{~mm}$ (Fig. 3(d)) and the mode is photon-like (Fig. 2). Beyond this position, $\lambda_{\mathrm{SW}}$ gradually decreases ( $n$ increases) until some minimum value is reached. This recorded minimum occurs at $x=70 \mathrm{~mm}$ and $135 \mathrm{~mm}$ on samples A and B, respectively (Figs. 3(a), 3(b), and $3(\mathrm{e}))$. While the measured amplitude of the surface wave fields also reduce to the noise floor at a similar position (i.e., propagation of the mode is forbidden), the phase data are more resilient at low signal levels and is less sensitive to variations in the background signal level. The phase information can, therefore, be used to pinpoint the trapping location of the surface mode more precisely.

Note that on approach to the trapping location modal index increases (Fig. 3(f)) but is limited by the Brillouin zone boundary because the surface wave wavelength becomes comparable to the pitch of the supporting structure (Fig. 3(a)). At this condition the surface wave is subject to Bragg reflections due to this photonic effect. In addition, our probe is no longer able to spatially resolve the phase in this limit due to its finite scattering cross section, and the phase does not reach $\pm \pi$. The observant reader will note that, after this position, the periodicity of the phase data recovers its free space value, a subject addressed in due course.

Also apparent in Figs. 3(a) and 3(b) is that the trapping position occurs at a distance along the sample beyond that which we would expect from our previous numerical modelling. Experimentally (Figs. 3(a) and 3(b)) we observe this to occur at $x=70 \mathrm{~mm}$ and $x=135 \mathrm{~mm}$ along samples $\mathrm{A}$ and $\mathrm{B}$, respectively, for the $20 \mathrm{GHz}$ component of the surface wave. In both cases, the trapping position corresponds to a lateral cavity width of $L=18.0 \mathrm{~mm}$. However, numerical predictions of the limiting frequency (inset of Fig. 2) suggest that this limit should occur on a region of the waveguide corresponding to $L=16.8 \mathrm{~mm}$. Additional modelling (not shown) suggests that while there is a small extension of the local field amplitude due to near field coupling between the detecting probe and the propagating surface wave, it is too small a 
contribution to completely account for this discrepancy. Therefore we propose that this discrepancy is associated with the intrinsic width of the mode itself. This conclusion is supported by the observation that the spatial separation of cavities corresponding to $L=16.8 \mathrm{~mm}$ and $L=18.0 \mathrm{~mm}$ is different for the two tapered samples. The finite width of the mode can be attributed to radiative decay channels (scattering of the mode out of the surface due to mode shape mismatch, Bragg reflection and coupling to the probe) and loss due to Ohmic heating of the metal.

Consider next the oscillations in the local field amplitude data (Fig. 3). They arise from a forward propagating surface wave mixing with a reflected mode, to produce an oscillation with period of $\lambda_{\mathrm{sw}} / 2$. This reflection is due to Bragg reflection and also a mismatch in the overlap of the fields of the surface wave, which is more significant for the shorter taper with a larger gradient $(\Delta L / \Delta x)$. The amplitude of these oscillations is indicative of the strength of reflection experienced by the surface mode. ${ }^{20}$ Therefore by inspection of Figs. 3(a) and 3(b) it is clear that a $20 \mathrm{GHz}$ surface wave supported by sample A experiences a reflection amplitude that is over twice that of a surface wave supported by sample $\mathrm{B}$. This is unsurprising as the rate of change of limiting frequency and modal index in sample A occurs spatially at more than twice the rate of sample B.

Now inspect the local field amplitude and phase of the surface wave after the trapping point is observed (Fig. 3). Quite unexpectedly, the spatial periodicity of the oscillations in the phase recovers their free space value $(n=1)$ whereas we would expect there to be no data beyond this point since the forward propagation of the fundamental mode is forbidden. Fig. 4(a) shows an experimental field plot in the $x y$ plane just above the top of the cavities and clearly illustrates that there exists a mode further along the array (Fig. 4(b)) with higher quantisation in lateral width (three antinodes). This is a surface wave associated with the lowest order Fabry Perot resonance of the $\mathrm{TE}_{03}$ waveguide mode in the cavities, ${ }^{36}$ with a limiting frequency greater than that of the original $\mathrm{TE}_{01}$ mode. It is excited by fields scattered into free space at the original trapping point (Fig. 4(c)), and therefore both the fundamental and the higher order surface mode of the array can be observed at the same fixed frequency. It should be noted that this multimodal excitation requires the symmetry of the modal fields to match that of the sample, and therefore surface modes associated with even lateralquantisations of the waveguide mode (e.g., $\mathrm{TE}_{02}$ ) cannot be excited. The excitation of the surface wave associated with the $\mathrm{TE}_{05}$ waveguide is also apparent from inspection of the amplitude data from sample A in Fig. 4(b) at $x=163 \mathrm{~mm}$.

In conclusion, the spatial dependence of the group velocity associated with a microwave surface wave supported across a metasurface with a graded geometry is studied. The metasurface consists of an array of closed-sided rectangular cavities obtruding from a ground plane whose lateral widths are increased as a function of distance from the point of excitation. This variation in cavity width results in a spatially dependent modal index. Subsequently, frequency components of the surface wave are slowed and trapped at different positions along the tapered metasurface. The trapping position and the behaviour of the supported mode approaching this

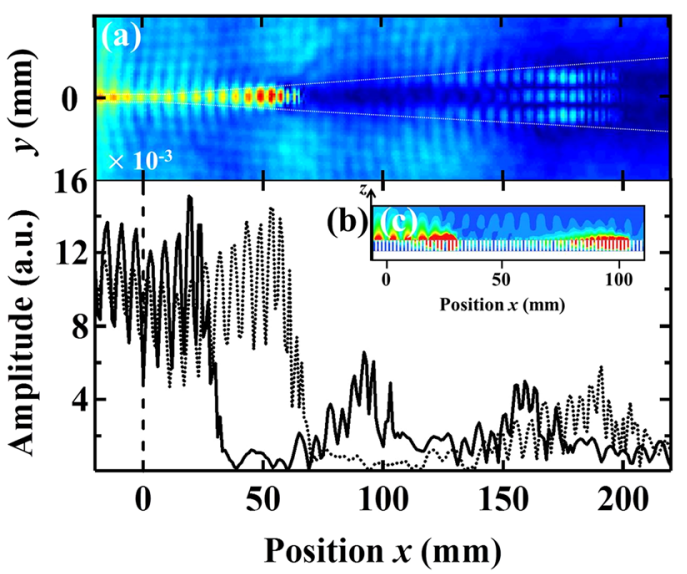

FIG. 4. (a) Experimental field plot in the $x-y$ plane (i.e., top down view) of electric-field amplitude at $25 \mathrm{GHz}$ on sample B. White dashed line indicates the edge of sample B. (b) Experimental observations of local field amplitude of the surface wave at $25 \mathrm{GHz}$ as a function of distance $x$ along samples A (solid black line) and B (dotted black line). Vertical black dashed line indicates the position of $L_{\text {Start }}$. Both (a) and (b) are at the same fixed height above the sample, $0.25 \mathrm{~mm}$. (c) Predictions of time averaged electric-field of the supported mode at $25 \mathrm{GHz}$ on sample $\mathrm{A}$ in the $x$-z plane; note the reradiation of the lowest order mode. Regions of high and low field are red and blue, respectively, for (a) and (c). Field predictions are obtained from FEM numerical modelling ${ }^{33}$ of the entire geometry of sample A, including a vertically orientated antenna providing near field excitation of the surface wave. The model geometry is surrounded by absorbing ("radiation") boundaries positioned over one wavelength away from the tapered cavity structure in the $x z$-planes and used as termination boundaries in the $y z$-planes, similar to the microwave foam absorber in experiment.

location have been experimentally observed with both local field amplitude and phase data. Four notable aspects of this data have been discussed. Primarily, the phase of the electric-field data provides a more accurate identification of the trapping location compared to amplitude measurements alone. Second, the exact stopping location of the surface wave does not correspond with numerical predictions obtained for a fixed-width one-dimensional array of cavities. Third, the oscillations observed in the local amplitude of the surface wave provide insight into the magnitude of reflection experienced by the surface mode close to this point. The origin of the reflection mechanism is considered. Finally, higher order lateral quantisations of the surface mode have been experimentally observed excited by scatter of the fundamental mode from the trapping point.

We would like to thank our sponsors, EPSRC, for their financial support and members of the Electromagnetic and Acoustic Materials Group at the University of Exeter, particularly Professor Euan Hendry, for useful discussions which helped to complete this work.

${ }^{1}$ F. Krauss, Nat. Photonics 2(8), 448 (2008).

${ }^{2}$ L. V. Hau, S. E. Harris, Z. Dutton, and C. H. Behroozi, Nature (London) 397, 594 (1999).

${ }^{3}$ K. L. Tsakmakidis, A. D. Boardman, and O. Hess, Nature 450, 397 (2007).

${ }^{4}$ M. S. Jang and H. Atwater, Phys. Rev. Lett. 107, 207401 (2011).

${ }^{5}$ S. Savo, B. D. F. Casse, W. Lu, and S. Sridhar, Appl. Phys. Lett. 98, 171907 (2011).

${ }^{6}$ W. T. Lu, Y. J. Huang, B. D. F. Casse, R. K. Banyal, and S. Sridhar, Appl. Phys. Lett. 96, 211112 (2010).

${ }^{7}$ J. Park, K.-Y. Kim, I.-M. Lee, H. Na, S.-Y. Lee, and B. Lee, Opt. Express 18, 598 (2010). 
${ }^{8}$ M. Santagiustina, G. Eisenstein, L. Thévenaz, J. Capmany, J. Mork, J. P. Reithmaier, A. De Rossi, S. Sales, K. Yvind, S. Combrié, and J. Bourderionnet, IEEE Photon. Soc. Newsletter 26, 5 (February 2012).

${ }^{9}$ H. Gersen, T. Karle, R. Engelen, W. Bogaerts, J. Korterik, N. van Hulst, T. Krauss, and L. Kuipers, Phys. Rev. Lett. 94, 073903 (2005).

${ }^{10}$ S. Ha, M. Spasenović, A. A. Sukhorukov, T. P. White, C. M. de Sterke, L. Kuipers, T. F. Krauss, and Y. S. Kivshar, J. Opt. Soc. Am. B 28, 955 (2011).

${ }^{11}$ Y. A. Vlasov, M. O’Boyle, H. F. Hamann, and S. J. McNab, Nature 438, 65 (2005).

${ }^{12}$ M. Spasenović, T. P. White, S. Ha, A. A. Sukhorukov, T. Kampfrath, Y. S. Kivshar, C. M. de Sterke, T. F. Krauss, and L. K. Kuipers, Opt. Lett. 36, 1170 (2011).

${ }^{13}$ J. D. Joannopoulos, S. G. Johnson, J. N. Winn, and R. D. Meade, Photonic Crystals: Molding the Flow of Light, 2nd ed. (Princeton University Press, Princeton, NJ, 1995).

${ }^{14}$ W. Barnes, A. Dereux, and T. Ebbesen, Nature 424, 824 (2003).

${ }^{15}$ M. Sandtke and L. Kuipers, Nat. Photonics 1, 573 (2007).

${ }^{16}$ E. Ozbay, Science 311(5758), 189 (2006).

${ }^{17}$ A. Karalis, E. Lidorikis, M. Ibanescu, J. Joannopoulos, and M. Soljačić, Phys. Rev. Lett. 95, 063901 (2005).

${ }^{18}$ Q. Gan, Y. Ding, and F. Bartoli, Phys. Rev. Lett. 102, 056801 (2009).

${ }^{19}$ M. Stockman, Phys. Rev. Lett. 93, 137404 (2004).

${ }^{20}$ S. He, Y. He, and Y. Jin, Sci. Rep. 2 , 583 (2012).

${ }^{21}$ J. B. Pendry, L. Martín-Moreno, and F. J. Garcia-Vidal, Science (N.Y.) 305, 847 (2004).
${ }^{22}$ S. Maci and G. Minatti, IEEE Trans. Antennas Wireless Propag. Lett. 10, 1499 (2011).

${ }^{23}$ H. M. Barlow and A. L. Cullen, IEEE Trans. Microwave Theory Tech. 100, 329 (1953).

${ }^{24}$ J. Suckling, A. Hibbins, M. Lockyear, T. Preist, J. Sambles, and C. Lawrence, Phys. Rev. Lett. 92, 147401 (2004).

${ }^{25}$ R. E. Collin, Field Theory of Guided Waves, 2nd ed. (McGraw-Hill, New York, 1991).

${ }^{26}$ E. M. G. Brock, E. Hendry, and A. P. Hibbins, Appl. Phys. Lett. 99, 051108 (2011).

${ }^{27}$ H. J. Rance, I. R. Hooper, A. P. Hibbins, and J. R. Sambles, Appl. Phys. Lett. 99, 181107 (2011).

${ }^{28}$ A. P. Hibbins, B. R. Evans, and J. R. Sambles, Science (N.Y.) 308, 670 (2005).

${ }^{29}$ D. Sievenpiper, R. F. J. Broas, N. G. Alexopolous, and E. Yablonovitch, IEEE Trans. Microwave Theory Tech. 47, 2059 (1999).

${ }^{30}$ M. Qiu, Opt. Express 13, 7583 (2005).

${ }^{31}$ Z. Ruan and M. Qiu, Appl. Phys. Lett. 90, 201906 (2007).

${ }^{32}$ E. Hendry, A. Hibbins, and J. Sambles, Phys. Rev. B 78, 235426 (2008).

${ }^{33}$ ANSOFT HFSS $^{\mathrm{TM}}$, Ansoft Corporation, Pittsburgh, PA.

${ }^{34}$ J. Zhu, Y. Chen, X. Zhu, F. J. Garcia-Vidal, X. Yin, W. Zhang, and X. Zhang, Sci. Rep. 3, 1728 (2013).

${ }^{35}$ Y. J. Zhou and T. J. Cui, Appl. Phys. Lett. 99, 101906 (2011).

${ }^{36}$ J. D. Edmunds, E. Hendry, A. P. Hibbins, J. R. Sambles, and I. J. Youngs, Opt. Express 19, 13793 (2011). 